For Table of Contents Use Only

Molecular Level Structures of Poly(n-Alkyl Methacrylate)s with Different Side Chain Lengths at the Polymer/Air and Polymer/Water Interfaces

Matthew L. Clarke, Chunyan Chen, Jie Wang, and Zhan Chen*

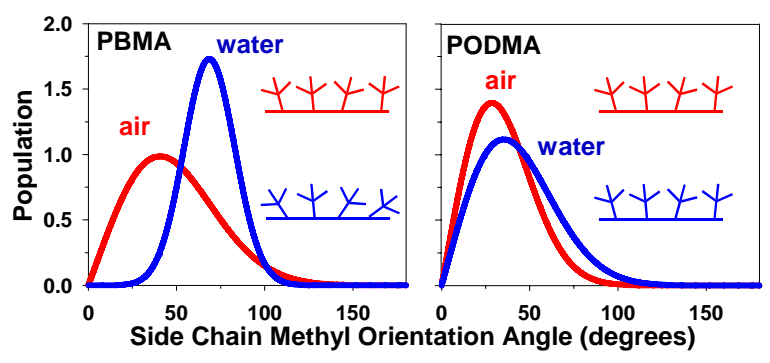




\title{
Molecular Level Structures of Poly(n-Alkyl Methacrylate)s with Different Side Chain Lengths at the Polymer/Air and Polymer/Water Interfaces
}

\author{
Matthew L. Clarke, Chunyan Chen, Jie Wang, and Zhan Chen* \\ Department of Chemistry, University of Michigan, Ann Arbor, Michigan 48109
}

*To whom all correspondence should be addressed.

Email: zhanc@umich.edu Fax: 734-647-4865

\begin{abstract}
Sum frequency generation (SFG) vibrational spectroscopy has been successfully applied to study molecular structures of several poly (n-alkyl methacrylate)s (PAMA)s with different side chain lengths at the PAMA/air and PAMA/water interfaces. We have observed that the ester side chains from all PAMAs always dominate the interface, but the orientation information of the methyl end group on the side chains vary, depending on the length of the side chain. The gauche defectscontributions from methylene groups on the side chains have been evaluated, and the surface structures have been related to the surface tension of these polymers. Different water restructuring behaviors have been observed for different PAMAs. This phenomenon and its reversibility are strongly dependent on the glass transition temperature of each polymer, which is influenced by the side chain length. Detailed data fitting and analysis has been discussed.
\end{abstract}




\section{Introduction}

Poly(alkyl methacrylate)s (PAMA)s have been used as very important polymer materials for biomedical applications. While bulk polymeric properties most obviously have a role in the usefulness of a polymer in biomedical applications, surface specific properties also play an important role in determining whether a material is a suitable biomaterial. ${ }^{1-10}$ The surface/interface properties of these polymers depend critically upon the molecular structure at the polymer surface or polymer/water interface. $^{11-16}$ To control polymer surface/interface properties by manipulating surface/interface structures, we must understand the detailed correlations between properties and structures of the polymer surfaces/interfaces. Ideally, the techniques used to characterize the polymer surface structures at the molecular level should be sensitive to molecular features, such as conformation, orientation, and chemical bonding. Since changes in these features are defined by subtle differences in the energy levels of the structures, highly sensitive molecular level spectroscopic signals such as vibrational spectra are needed for characterization.

After Langmuir's results on surface restructuring in water, ${ }^{17}$ much research has been undertaken to understand polymer surface dynamics and structures in water. Most surface science techniques are operable only with the sample under ultrahigh vacuum. ${ }^{18,19}$ While some of these techniques, such as secondary ion mass spectrometry (SIMS), can capture molecular level information, they cannot characterize the surface in a hydrated state. Freeze-drying x-ray photoelectron spectroscopy (XPS) has been developed to study polymer surface restructuring in water, but it cannot provide orientation information of surface functional groups. ${ }^{20}$ Contact angle measurements show that many polymer

surfaces change when exposed to water, ${ }^{21-24}$ but they cannot provide a molecular level picture of how such changes occur. 
Recently, SFG has been developed into a powerful technique to investigate surface structures of polymer materials in different environments. ${ }^{25-39}$ SFG signal can only be generated from media that does not have inversion symmetry (under the electric-dipole approximation), thus most bulk materials will not generate SFG signal since they possess inversion symmetry. Our previous SFG research has shown that surface restructuring behaviors of various polymers in water are very different. ${ }^{32,37} \mathrm{~A}$ method was also developed to quantify the orientation of the functional groups on polymer surfaces in air and water. ${ }^{33}$ Here, we will continue to investigate molecular surface structures of PAMAs in further depth and correlate such molecular surface structures to their physical properties, such as surface tension. Surface/interfacial structures of various polymethacrylates and polyacrylates have been studied

using SFG. ${ }^{32,33,37,40-47}$ In this research, after investigating polymer surfaces in air, SFG will be applied to monitor surface structures of PAMAs in contact with water. A possible relation between the water restructuring behaviors of different PAMAs and the difference in mobility of these surface molecules will be established.

\section{Experimental}

\subsection{Sample preparation}

All hydrogenated polymers were purchased from Scientific Polymer Products Inc. and used as received. The basic molecular structure for the PAMAs is presented in Figure 1. Polymer films were prepared by spin-coating $2 \mathrm{wt} \%$ polymer/toluene solution onto fused-silica windows ( 1 inch diameter, $1 / 8$ inch thick, ESCO Products Inc.). All spin-cast samples were oven dried at $80{ }^{\circ} \mathrm{C}$ for $24 \mathrm{~h}$ before analysis. Polymer films were approximately $100 \mathrm{~nm}$ thick.

\subsection{SFG Instrumentation and Orientation Analysis}


SFG spectra were collected using an EKSPLA system (Vilnius, Lithuania). Specifics about our system have been described in our previous publications. ${ }^{32,48}$ In this work, SFG spectra with different polarization combinations, ssp (s-polarized SF output, s-polarized visible input, and p-polarized infrared input) and sps, were collected.

The orientation information of the methyl groups can be evaluated by measuring the value of $\left|\chi_{y y z, a s}\right| /\left|\chi_{y z y, a s}\right|,{ }^{36,37}$ which can be obtained by fitting SFG spectra using eq 1 . The tensor component of the measured nonlinear susceptibility $\chi^{(2)}$, e.g., $\chi_{\text {ssp }}^{(2)}$, can be written as:

$$
\chi_{\mathrm{s}, \mathrm{sp}}^{(2)}=\chi_{\mathrm{R}, \mathrm{spp}}^{(2)}+\chi_{\mathrm{NR}, \mathrm{ssp}}^{(2)}=\sum_{q} \frac{A_{q, y y z}}{\omega_{\mathrm{IR}}-\omega_{q}+i \Gamma_{q}}+\chi_{\mathrm{NR}, \mathrm{yyz}}^{(2)}
$$

where $\chi_{\mathrm{NR}}{ }^{(2)}$ is the non-resonant background from the substrate and $A_{q, y y z}, \omega_{\mathrm{IR}}, \omega_{q}, \Gamma_{\mathrm{q}}$ are strength of the $q$ th vibrational mode, frequency of the infrared beam, resonance frequency and damping constant of the $q$ th vibrational mode, respectively. ${ }^{33,37}$ The orientation of surface functional groups can be determined by their ssp and sps SFG spectra. As described in the literature, the different components of $\chi^{(2)}$ (in the lab coordinate system) are related to different components of the molecular hyperpolarizability $\beta^{(2)}$ (in the molecular coordinate system) by the orientation angles of these functional groups. ${ }^{38,39,49}$ We can deduce the orientation information of each functional group after measuring their different components of $\chi^{(2)}$ by fitting the SFG spectra using eq 1 and knowing their molecular hyperpolarizabilities $\beta^{(2)} \cdot{ }^{39}$ Assuming that methyl groups have $\mathrm{C}_{3 \mathrm{v}}$ symmetry, the relationship between different components of the second-order nonlinear susceptibility $\chi^{(2)}$ in the lab coordinate system and the molecular hyperpolarizibility $\beta^{(2)}$ in the molecular coordinate system can be established. ${ }^{39,48}$ Here only the relations deduced from ssp and sps SFG spectra for the asymmetric stretch of methyl groups are listed below. 


$$
\begin{aligned}
& \chi_{y y z, a s}^{(2)}=\chi_{x x z, a s}^{(2)}=-\frac{1}{2} N_{s} \beta_{c a a}^{(2)}\left(<\cos \theta>-<\cos ^{3} \theta>\right) \\
& \chi_{z x x, a s}^{(2)}=\chi_{z y y, a s}^{(2)}=\chi_{y z y, a s}^{(2)}=\chi_{x z x, a s}^{(2)}=\frac{1}{2} N_{s} \beta_{c a a}^{(2)}<\cos ^{3} \theta>
\end{aligned}
$$

To deduce the orientation angle of the methyl group, it is more convenient to measure the ratios of independent non-vanishing $\chi^{(2)}$ components so that the absolute determination of $N_{\mathrm{s}}$ and $\beta^{(2)}$ does not have to be found. ${ }^{39}$

Therefore, we have:

$$
\left|\frac{\chi_{y y z, a s}}{\chi_{y z y, a s}}\right|=\left|\frac{<\cos \theta>-<\cos ^{3} \theta>}{<\cos ^{3} \theta>}\right|
$$

The bracket $<>$ implies averages of tilting angles. For example,

$$
\begin{aligned}
& <\cos ^{n} \theta>=\int_{0}^{\pi} \cos ^{n} \theta f(\theta) \sin \theta d \theta \\
& \text { and } f(\theta)=C \exp \left[-\left(\theta-\theta_{0}\right)^{2} / 2 \sigma^{2}\right]
\end{aligned}
$$

where $f(\theta)$ is a Gaussian function describing the angle distribution, $C$ is the normalization constant, $\theta_{0}$ is related to the average orientation angle of the methyl group vs the surface normal and $\sigma$ is the angle distribution parameter.

\subsection{Surface Tension Measurements}

Based on the values of the contact angle between a polymer surface and water as well as another liquid - diiodimethane - the surface tension of the polymer film can be calculated using the Geometric function (eqs 7 and 8). The values of $\gamma^{\mathrm{d}}$ and $\gamma^{\mathrm{p}}$ for water are 21.8 and $51.0 \mathrm{dyn} / \mathrm{cm}$ and those for diiodimethane are 49.5 and $1.3 \mathrm{dyn} / \mathrm{cm}$, respectively. ${ }^{10,11}$ The contact angles were measured using a KSV contact angle goniometer.

$$
\left(1+\cos \theta_{w}\right) \gamma_{w}=2\left[\left(\gamma_{w}^{d} \gamma_{s}^{d}\right)^{1 / 2}+\left(\gamma_{w}^{p} \gamma_{s}^{p}\right)^{1 / 2}\right]
$$




$$
\left(1+\cos \theta_{m}\right) \gamma_{m}=2\left[\left(\gamma_{m}^{d} \gamma_{s}^{d}\right)^{1 / 2}+\left(\gamma_{m}^{p} \gamma_{s}^{p}\right)^{1 / 2}\right]
$$

\section{Results and Discussion}

\subsection{Optimizing the Polymer Film Thickness}

For a typical polymer thin film coated on a fused silica substrate, there are two different interfaces: the polymer/substrate and polymer/air interfaces. In our previous publications, we have confirmed that the SFG signals collected from the polymer thin film are mainly contributed by the

polymer/air interface. ${ }^{32,33,48}$ The contribution from the polymer/substrate interface is fairly small and negligible. However, for a more quantitative analysis of the polymer surface structures, the effect of SFG signals from the polymer/substrate interface on the overall signals obtained has to be considered. Thickness-dependent interference effects between the polymer/substrate and the polymer/air interfaces have previously been reported. ${ }^{34,50-52}$ We have collected spectra from PBMA films of varying thickness, and have observed a thickness-dependence of the SFG spectra. Additionally, through calculations based on our experimental results, we found that the interference from the polymer/substrate interface can be minimized by selecting an optimal film thickness. ${ }^{53}$ Certain film thicknesses generate SFG signals where the substrate/polymer and polymer/contact medium interfaces are maximally constructive or maximally destructive. At an intermediate film thickness, where the SFG signal is the average of these two extreme cases, the weaker signal generated from the substrate/polymer interface will be negligible, and the detected signal can be considered to be solely from the polymer/contact medium interface. For more details see the Supporting Information. In this paper, all spectra shown are collected from the polymer film by choosing an appropriate thickness, and, therefore, the effect of signals from the polymer/substrate interface can be neglected during quantitative analysis. 


\section{2 Molecular Structures at the PAMA/Air Interfaces}

To elucidate the effect of side chain length on the polymer surface structures in detail, we have investigated and compared surface structures of several PAMAs with side chains varying from methyl to octadecyl groups in air and in water. We will first discuss surface structures of these PAMAs in air based on the SFG spectra (Figure 2).

Both ssp and sps SFG spectra are shown in Figure 2 for each PAMA. Generally, the ssp spectra are mainly contributed by the symmetric stretches of the functional groups on the side chains of each polymer, while the sps spectra are mainly contributed by the asymmetric stretches of these groups. ${ }^{33,48}$ The peak positions for the symmetric and asymmetric stretches of the methyl groups on the ester side chains for different PAMAs vary because the methyl groups can have different chemical environments (i.e., different distances from the ester group). When the side chain length is equal to or larger than 4 carbons ( $\mathrm{m}=4,6,8,12$, and 18$)$, all SFG spectra are mainly composed of five peaks: $\mathrm{CH}_{2}$ symmetric stretch at $2850 \mathrm{~cm}^{-1}, \mathrm{CH}_{3}$ symmetric stretch at $2875 \mathrm{~cm}^{-1}, \mathrm{CH}_{2}$ asymmetric stretch at $2920 \mathrm{~cm}^{-1}$, Fermi resonance at $2940 \mathrm{~cm}^{-1}$, and $\mathrm{CH}_{3}$ asymmetric stretch at $2960 \mathrm{~cm}^{-1}$. The features of these spectra resemble those from normal alkyl chains. ${ }^{20}$ The methyl groups on the backbone ( $\alpha$-methyl groups) have characteristic peak positions: symmetric stretching at $\sim 2930 \mathrm{~cm}^{-1}$, and asymmetric at $\sim 2990 \mathrm{~cm}^{-1}$. Such signals were not observed in the SFG spectra nor in spectral fitting, showing that they do not have substantial surface coverage. ${ }^{33}$ However, when the side chain length is shorter than 4 carbons $(\mathrm{m}=1,2$, and 3), the peaks for the vibrational modes of the functional groups on the side chains shift slightly compared to those in PAMAs with $m \geq 4$, because such modes from PAMAs with shorter chains can be perturbed by the ester group. The perturbation depends on the distance between the ester group and the methylene or methyl groups. When the side chain is long enough (i.e., $\mathrm{m} \geq 4$ ) such a perturbation is too small to be noticed. The methylene SFG signal also depends on the side chain length of the PAMAs. 
When $\mathrm{m} \geq 4$ the normal methylene groups dominate the spectra. When the chain is short, the methylene group next to the oxygen (ester group) may contribute to the signal and complicate the spectra.

The peak assignments for PMMA have been discussed in detail in the literature. ${ }^{23}$ Generally, the SFG spectra (ssp and sps) for PMMA are dominated by the symmetric $\left(2955 \mathrm{~cm}^{-1}\right)$ and asymmetric stretches (2990 and $3016 \mathrm{~cm}^{-1}$ ) of the ester methyl groups. Signals from the methyl groups on the polymer backbone are much weaker. ${ }^{48}$ The peak assignments for the SFG spectra collected from the PEMA surface have been discussed in our recent publication. ${ }^{37}$ We assign the peaks at $2935 \mathrm{~cm}^{-1} / 2870$ $\mathrm{cm}^{-1}$ (ssp spectrum) to the symmetric C-H stretch/Fermi resonance of the methyl group at the ester ethyl end. Two small peaks around $2900 \mathrm{~cm}^{-1}$ and $2955 \mathrm{~cm}^{-1}$ in the ssp SFG spectra are assigned to the vibrational modes of the symmetric and asymmetric stretches of methylene groups, respectively. ${ }^{54}$ The sps spectrum is dominated by the asymmetric stretch of the side chain methyl group at $2975 \mathrm{~cm}^{-1} .54,55$ Using partially deuterated PEMA samples, we showed that the $\alpha$-methyl groups do not contribute to SFG signals. For PPMA, the SFG spectra ( $s s p$ and sps) have very similar features to those of PBMA, but a slight shift was found (by $\sim 5 \mathrm{~cm}^{-1}$ ) for some of the major vibrational modes (e.g., for $\mathrm{CH}_{3}$ symmetric stretch, Fermi resonance and $\mathrm{CH}_{3}$ asymmetric stretches).

According to the SFG spectra in Figure 2, we can draw the conclusion that all PAMA surfaces are primarily covered by the ester side chains because these SFG spectra are dominated by the vibrational modes from the methylene and methyl groups on the ester side chains. The effect of side chain length on the conformations of these alkyl side chains can be clearly observed based on these SFG spectra. For example, when the side chain is short, $(\mathrm{m} \leq 4)$, all ssp spectra are dominated by the symmetric stretch of the methyl end group and sps spectra are dominated by the asymmetric stretch of the methyl end group. When the side chain length becomes longer $(\mathrm{m}>4)$, the contribution from the symmetric stretch of methylene groups becomes stronger as the side chain length increases. This 
indicates that more and more gauche defects are detected on the PAMA surfaces in air. These effects induced by side chain length have been observed on other alkyl chains..$^{30,56,57}$ To quantify the surface structures of PAMAs in air, we have fitted all the spectra. After calculating the values of $\left|\chi_{y y z, a s}\right| /\left|\chi_{y z y, a s}\right|$ for each polymer, we have plotted $\left|\chi_{y y z, a s}\right| /\left|\chi_{y z y, a s}\right|$ vs the chain length (Figure 3a). The results from the polymer/water interface are also presented and will be discussed later. To demonstrate how the orientation of a functional group is related to the value of $\left|\chi_{y y z, a s}\right| /\left|\chi_{y z y, a s}\right|$, we have calculated the value of $\left|\chi_{y y z, a s}\right| /\left|\chi_{y z y, a s}\right|$ as a function of $\theta_{0}$ for several orientation angle distributions $\left(\sigma=0^{\circ}, 10^{\circ}, 20^{\circ}\right.$, $\left.30^{\circ}, 40^{\circ}, 50^{\circ}, 90^{\circ}\right)$ as shown in Figure 4. A fixed value of $\left|\chi_{y y z, a s}\right|\left|\chi_{y y, a s}\right|$ corresponds to various possible combinations of $\theta_{0}$ and $\sigma$. Generally, different tilting angles $\theta_{0}$ depend on different values of $\left|\chi_{y y z, a s}\right| /\left|\chi_{y z y, a s}\right|$. When the ratio $\left|\chi_{y y z, a s}\right| /\left|\chi_{y z y, a s}\right|$ increases, the methyl groups tend to have an increasing average orientation angle $\theta_{0}$, which indicates that the methyl groups tend to tilt more toward the surface. Figure 3a shows that the measured values of $\left|\chi_{y y z, a s}\right| /\left|\chi_{y z y, a s}\right|$ for the methyl groups on all the PAMAs are centered on 0.5. Based on Figure 4, the methyl groups on all surfaces have an orientation angle smaller than $40^{\circ}$, demonstrating that all methyl groups tend to stand up at the surface. The slight difference of orientation angle of the methyl groups may be due to the different chain mobility of each polymer.

\subsection{Different Water Restructuring Behaviors at PAMAs/Water Interfaces}

We have also observed that PAMAs with different ester side chains have different surface restructuring behaviors. Early studies of the PAMA surfaces by dynamic contact angle indicate that there is a contact angle hysteresis when these polymers contact water. ${ }^{58}$ The mobility of the polymer surface chains and the reorientation of the surface functional groups might explain the hysteresis; 
however no direct evidence confirms this hypothesis due to lack of the suitable techniques. Herein, we have provided some direct evidence to support the early research.

The water restructuring behaviors of these PAMAs will be discussed in the order of the side chain length. For the shortest side chain $(\mathrm{m}=1)$, the SFG spectra from the PMMA/water interface resembles those from the PMMA/air interface (data not shown), which indicates that the surface dominating ester methyl groups on the PMMA surface do not change orientation upon contacting water. ${ }^{32}$ The intensity decrease observed in SFG signals of the PMMA/water interface is due to the different Fresnel coefficients. ${ }^{32,33}$ As the side chain length increases, the features of the SFG spectra collected from the PAMA/water interfaces change substantially from those collected from the PAMA/air interfaces. Qualitatively, it demonstrates that the surfaces of these PAMA $(m>1)$ films restructured in water. We noticed that for PAMAs with $m=2,3,4$ and 6, the ssp SFG spectra (Figure 5) collected after the surface is brought into contact with water is no longer dominated by the symmetric stretch of the methyl groups, but rather by the asymmetric stretch of the methyl groups. This indicates that the average orientation angle of the methyl end-groups on these polymer/water interfaces becomes larger, thus these groups tilt more toward the surface. ${ }^{24}$ Detailed analysis on the spectra from the PAMA/water interfaces will be discussed in the next section. All the polymer films shown in Figure 5 recover after removing these samples from water, indicating that the surface restructuring behaviors of these PAMAs ( $\mathrm{m}=2,3,4$, and 6$)$ in water are reversible. However, as the length of the side chain becomes longer (e.g., POMA with $\mathrm{m}=8$, and PLMA with $\mathrm{m}=12$ ), the PAMA surfaces lose order after contacting water, as evidenced by the loss of SFG from either the POMA/water or PLMA/water interface (data not shown). For these two polymers, upon removal from water, the SFG spectra cannot recover completely to those collected from the polymer/air interfaces before contacting water. This shows that the restructuring behaviors of these two polymer surfaces in water are irreversible. This 
behavior is similar to that observed for poly(ethyl acrylate). ${ }^{37}$ When the side chain length becomes even longer (PODMA with $\mathrm{m}=18$ ), SFG signal was detected from the PODMA/water interface. The spectral features have changed slightly compared with that from the polymer/air interface and the spectrum recovered after removal of water. This also demonstrates that the functional groups on the PODMA surface reorganize upon contacting water and this reorganization behavior is reversible. Therefore, we have observed that the water restructuring behavior of PODMA is similar to the polymers with shorter chains (such as PBMA), but not to the polymers with relatively longer chains (POMA and PLMA). PODMA is a semi-crystalline polymer and has a melting point of $37^{\circ} \mathrm{C}$, thus the packing of the long alkyl side chains stabilizes the polymer surface, and the PODMA surface still has some ordered structure in water. Therefore, the water restructuring behaviors of these seven PAMAs can be divided into four categories, as shown in Table 1, based on their restructuring behaviors in water.

\subsection{Detailed Orientation Analysis for PAMAs}

Measuring the absolute intensity of the SFG signal detected from the polymer surface can allow for some further details about the polymer surface to be elucidated. Previous research employed this method to deduce details about PBMA surface structural changes by monitoring the SFG absolute

intensity of the methyl symmetric stretch. ${ }^{33}$ Using the same method, we have analyzed the orientation of the methyl groups on PPMA, PBMA, PHMA, and PODMA in further depth. For the methyl mode:

$$
\chi_{y y z, s}^{(2)}=\frac{1}{2} N_{s} \beta_{c c c, s}^{(2)}\left[<\cos \theta>(1+r)-<\cos ^{3} \theta>(1-r)\right]
$$

where $r=\beta_{a a c, s}^{(2)} / \beta_{c c c, s}^{(2)}$. Our previous work has found $\mathrm{r}=1.8$, and $N_{s}$ to be $\sim 7.0 \times 10^{-14} \mathrm{~cm}^{-2}$ for PBMA. ${ }^{33}$ Here $N_{s}$ is the effective surface coverage, which contains effects from the Fermi resonance and local field correction factors. Eq 4 can be rewritten as: 
$\left|\frac{\chi_{y y z, a s}}{\chi_{y z y, a s}}\right|=\left|\frac{<\cos \theta>-<\cos ^{3} \theta>}{<\cos ^{3} \theta>}\right|=\left|\frac{1}{\gamma}-1\right|$

where $\gamma=\frac{<\cos ^{3} \theta>}{<\cos \theta>}$. Further, eq 9 can be rearranged to:

$\chi_{y y z, s}^{(2)}=\frac{1}{2} N_{s} \beta_{c c c, s}^{(2)}<\cos \theta>[1+r-\gamma(1-r)]$

Therefore, by deducing $\gamma$ from eq 11, and measuring the absolute intensity $\left|A_{y y z, s}\right|^{2}$, it is possible to calculate $<\cos \theta>$ and $<\cos ^{3} \theta>$.

It is vital to first select an appropriate $N_{s}$ for the calculations. It is expected that this number will be similar for the PAMA surfaces, but not identical. Since the methyl surface densities are not known, they will be estimated by calculation guided by our experimental results. The $\left|A_{y y z, s}\right|^{2}$ value of the methyl groups for each polymer is determined by fitting the spectra using eq 1 . The intensities of the signals collected from the polymer/water interface were adjusted to account for the difference in Fresnel factors between the air and water measurements. These intensity values are displayed in Figure $3 b$. Any effects from Fermi resonance on the methyl symmetric peak is believed to be similar in air and in water and is considered accounted for in the choice of the surface density. ${ }^{33}$ The absolute intensity for PBMA has previously been determined, ${ }^{33}$ and to simplify the calculations, the intensities used here will be relative to the PBMA methyl symmetric stretch intensity in air.

The relative absolute intensities in air and in water as a function of $\theta_{0}$ for different $N_{s}$ can be calculated, as was performed for PBMA in a previous publication. ${ }^{33}$ The $N_{s}$ can then be determined by using the measured intensity. In this calculation, it is necessary to determine an appropriate $\sigma$ (angle distribution) for each $\theta_{0}$. This is performed by using the determined $\left|\chi_{y y z, a s}\right| /\left|\chi_{y z y, a s}\right|$ ratio for that interface, and determining the $\theta_{0}, \sigma$ pairs using eq 4 . For PPMA it is observed that the measured relative 
intensity value crosses $N_{s}=5.5 \times 10^{-14} \mathrm{~cm}^{-2}$ for both the air and water measurements, and therefore this is assumed to be the surface coverage. Similar calculations show $N_{s}=6.0 \times 10^{-14} \mathrm{~cm}^{-2}$ for PHMA, and $7.0 \times 10^{-14} \mathrm{~cm}^{-2}$ for PBMA. The $N_{s}$ for PODMA was approximated to be $7.0 \times 10^{-14} \mathrm{~cm}^{-2}$. The $N_{s}$ for a polymer is assumed to be the same in air and in water.

The relative absolute intensity as a function of $\theta_{0}$, for different $\sigma$ and $\left|\chi_{y y z, a s}\right| /\left|\chi_{y z y, a s}\right|$ is displayed in Figure 6. To easily compare the different polymer films, the relative absolute intensities of PPMA, PHMA and PODMA have been adjusted for their different surface coverages using $I_{i j k} \propto\left|N_{s}\right|^{2}$. Experimental error can affect these results, as indicated by the shaded areas in Figure 6. However, this magnitude of error will not substantially change the conclusions. The Gaussian orientation distributions of the surface methyl groups deduced by SFG are presented in Figure 7 using the normalized distribution population $N_{s}(\theta)=N_{s} f(\theta) \sin (\theta)$. It is observed that the surface dominating methyl groups of these four polymer films orient towards the surface normal in air, all with $\theta_{0}<20^{\circ}$. The distributions for PPMA, PBMA and PHMA are very similar in air. PODMA has a narrower distribution centered more closely to the surface normal. In water PPMA, PBMA and PHMA all lie down more on the surface $\left(\theta_{0}>60^{\circ}\right)$ with a slightly narrower distribution than in air. As the side chain length increases, the methyl groups tend to lie down more on the surface with a narrower distribution. This is likely due to the ability of the longer side chain polymers to more fully reorient, due to their lower $T_{g}$. The PODMA surface is unique and undergoes little change upon exposure to water, likely stabilized by the semi-crystalline structure at the surface. It should be mentioned that error can occur in the data analysis and experimental procedures. Methods to analyze this error have been demonstrated in our previous research. ${ }^{33}$ Similar methods can be used to analyze the data here. 


\subsection{Relating Restructuring Behavior to PAMA Surface Tension and $T_{g}$}

To establish the surface structure-property relationship, the surface tension has been calculated based on eqs 7 and 8 . The surface tension of each PAMA and the measured contact angles in water and diiodimethane are listed in Table 1. When the side chain becomes shorter, the surface tension of the PAMAs increases. The more hydrophilic ester group plays an important role in this surface tension trend. The surface tension and $T_{g}$ both exhibit the same trend: they decrease as the side chain length increases.

If we compare the water restructuring behaviors of these polymers with the glass transition temperature of each polymer, a correlation could be established. PMMA has a very high $T_{\mathrm{g}}$, which suggests a more robust chain. In addition, the surface dominating ester methyl group is more "hydrophilic." Therefore, the PMMA surface does not restructure in water. For PAMAs with intermediate side chain length $(\mathrm{m}=2,3,4$, and 6$)$, they have moderate values of $T_{\mathrm{g}}$. Upon contacting water, the side chains are mobile enough to reorient, while the backbones are still robust and do not reorganize. Therefore, the surfaces are still ordered, although the orientation of the side chains changes.

The SFG spectra in Figure 5 were fitted and the values of $\left|\chi_{y y z, a s}\right| /\left|\chi_{y z y, a s}\right|$ have been calculated (Figure 3a). All values of $\left|\chi_{y y z, a s}\right| /\left|\chi_{y z y, a s}\right|$ for these five PAMA/water interfaces are much larger than those for the PAMA/air interfaces, indicating that the average orientation angles of the methyl groups on the polymer surface become larger and the methyl group tilt more toward the surface. The minor difference among these four PAMAs might be due to the slight difference in $T_{\mathrm{g}}$ of each polymer. For example, PHMA is softest among these four and the methyl groups tilt more toward the surface as indicated by a larger value of $\left|\chi_{y y z, a s}\right| /\left|\chi_{y z y, a s}\right|$. POMA and PLMA have much lower $T_{\mathrm{g}}$ values. Their 
surface molecules are actually very mobile; therefore, the backbones can reorganize upon contacting water. It is also noticed that all these two polymer surfaces become rough after contacting water. We have tested the restructuring behaviors of one thick POMA and PLMA film and discovered that the both surfaces lose order gradually. The time-dependent loss of SFG signal after POMA and PLMA films are brought in contact with water has been observed (data not shown), and are similar to what has been discovered for poly(ethyl acrylate) films in contact with water. ${ }^{37}$ We notice that both films become rough as they lose SFG signal. The time for the film to become totally rough matches very well with the time for the SFG signal to disappear, indicating that the loss of both POMA and PLMA surface order is a process of surface roughening. For films with different thicknesses, it takes different times to dewet from the substrate (nearly instantly for $100 \mathrm{~nm}$ films, and $\sim 10$ minutes for $200 \mathrm{~nm}$ films). This disorder is irreversible - even after curing the films above $T_{\mathrm{g}}$ the surfaces remain rough. As for PODMA, although the $T_{\mathrm{g}}$ is very low, it has a semi-crystalline structure at room temperature which is below its $T_{\mathrm{m}}$. Due to this structure, the polymer chain is more robust, only the side chains reorganize and they can recover upon the removal of water. Among these PAMAs, PODMA is the most hydrophobic, as revealed by a large contact angle. However, the water restructuring process of PODMA is still reversible, unlike POMA and PLMA. This leads us to believe that the chain mobility controls the water restructuring behaviors of these polymers more than the hydrophobicities of these polymers.

\section{Conclusion}

We have investigated surface structures of several PAMAs with different side chain lengths in air and in water. In air, PAMAs with long side chains have more gauche defects more methylene signals from the side chains on the surface. In water, the surface restructuring behaviors of PAMAs are strongly dependent on the chain mobility of these polymers, which are determined by the side chain length. For PMMA, no substantial surface restructuring of the surface dominating ester methyl groups was 
observed. For PAMAs with intermediate side chain lengths, surface dominating side chain end-groups change their orientations upon contacting water and such surface methyl orientation changes are reversible. When the polymer has an extremely low $T_{\mathrm{g}}$, such as PAMAs with long side chains, the polymer backbone on the surface can reorganize upon contacting water and then the surface becomes rough, losing structural order. Such a surface can be very rough so that the film completely dewets from the substrate. As a result, the SFG signal collected from such a polymer/water interface disappears. The timescale for such a process to occur depends on the thickness of the polymer film. We have shown that such surface restructuring in water is irreversible. However, when the side chain length continues to increase (i.e., PODMA), the water restructuring behavior becomes reversible again, because the surface is semi-crystalline and the surface structure is controlled by the very long chains rather than being influenced by the mobile backbone.

Acknowledgements This research is supported by the National Science Foundation (CHE-0315857 and CHE-0449469) and Office of Naval Research (Grant N00014-02-1-0832). M. C. was supported by a Rackham Predoctoral Fellowship from the University of Michigan. 


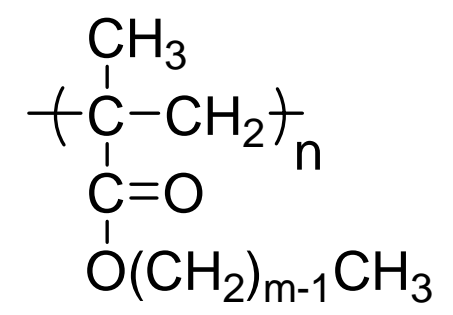

Figure 1. Structure of PAMAs with varying side chain length (m).
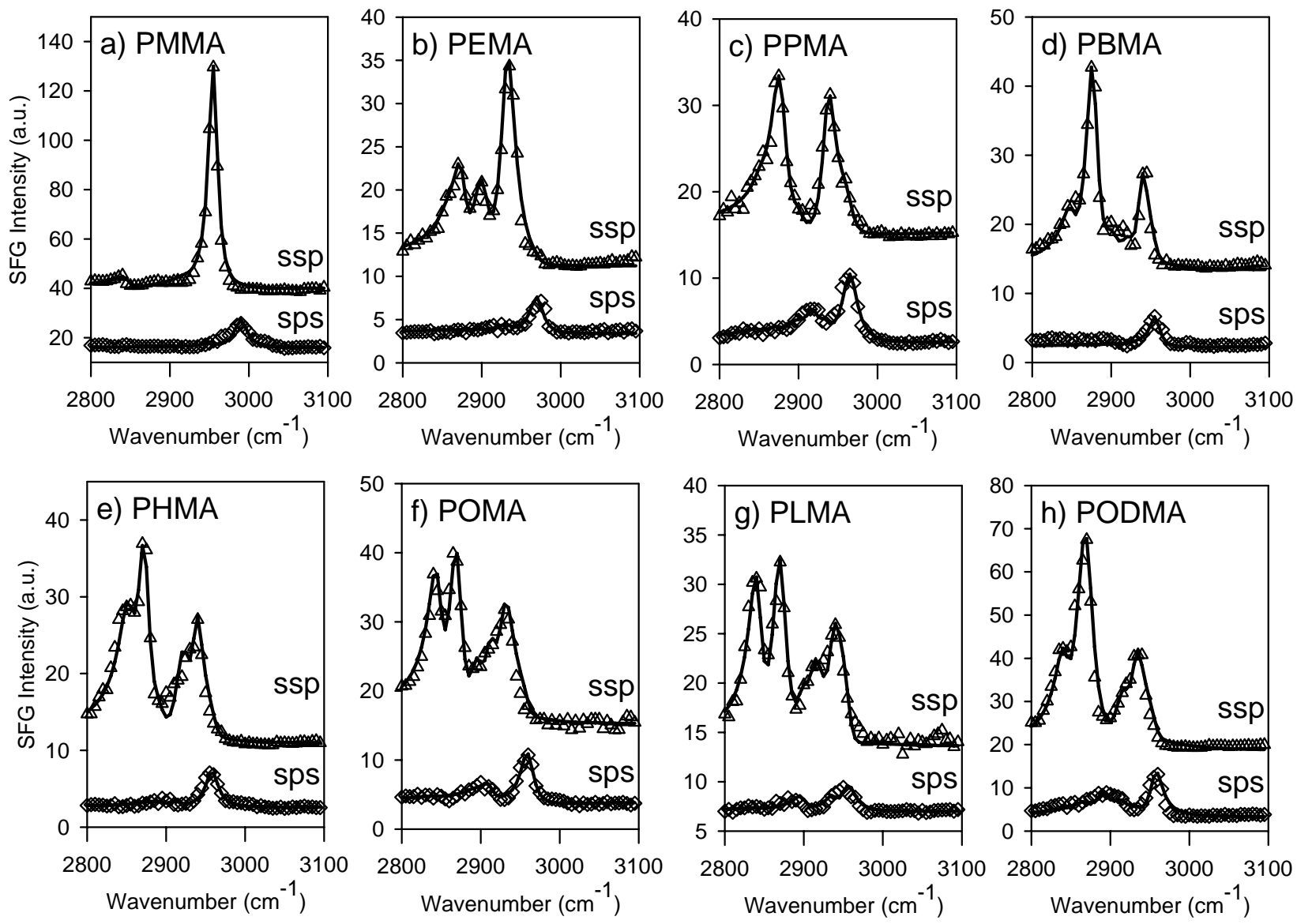

Figure 2. Measured (points) and fitted (lines) SFG spectra (ssp and sps) of PAMAs in air. 

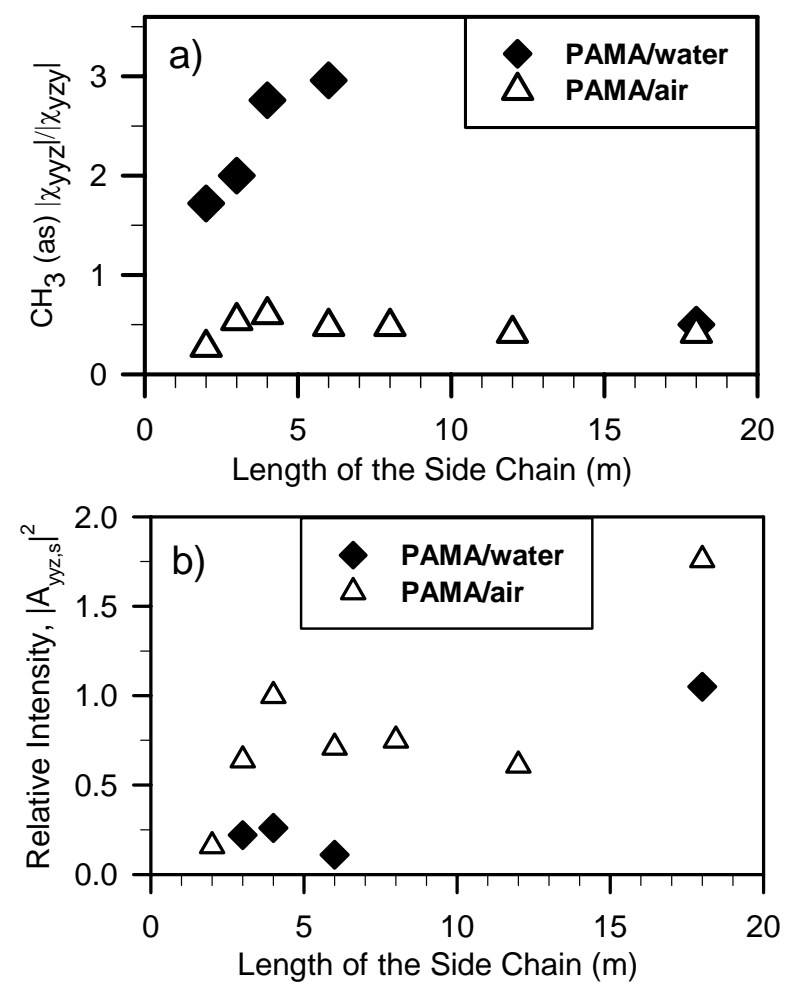

Figure 3. Measured values of a) $\left|\chi_{y y z, a s}\right| /\left|\chi_{y z y, a s}\right|$ for the methyl groups at the ester side chains and b) Relative intensity of the ssp methyl symmetric stretch of PAMAs in air and in water as a function of the length of the side chain. 


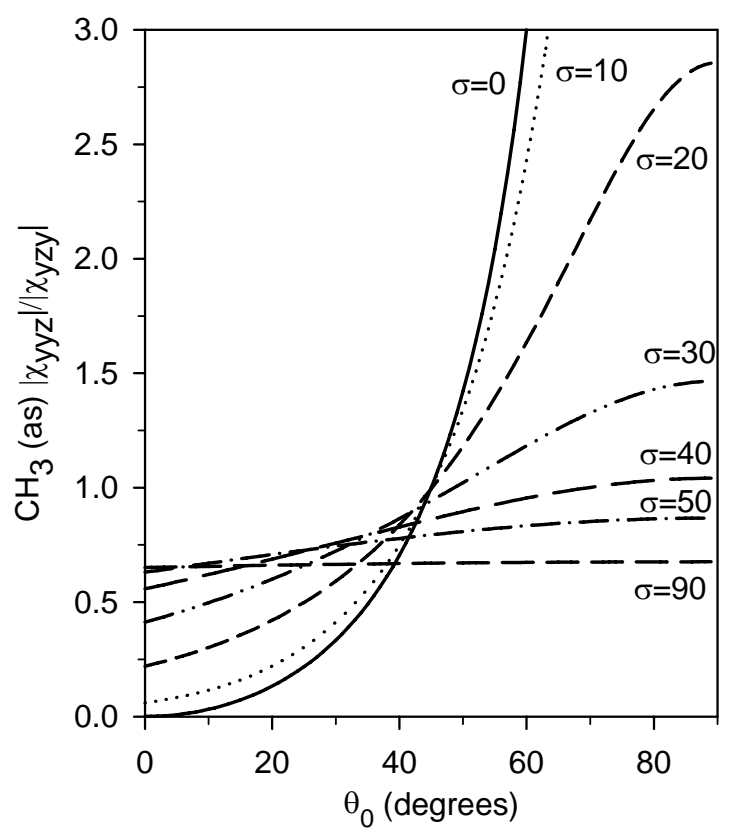

Figure 4. Calculated values of $\left|\chi_{y y z, a s}\right| /\left|\chi_{y z y, a s}\right|$ for the methyl groups at the ester side chains as a function of the tilting angle $\theta_{0}$ and angle distribution $\sigma$.
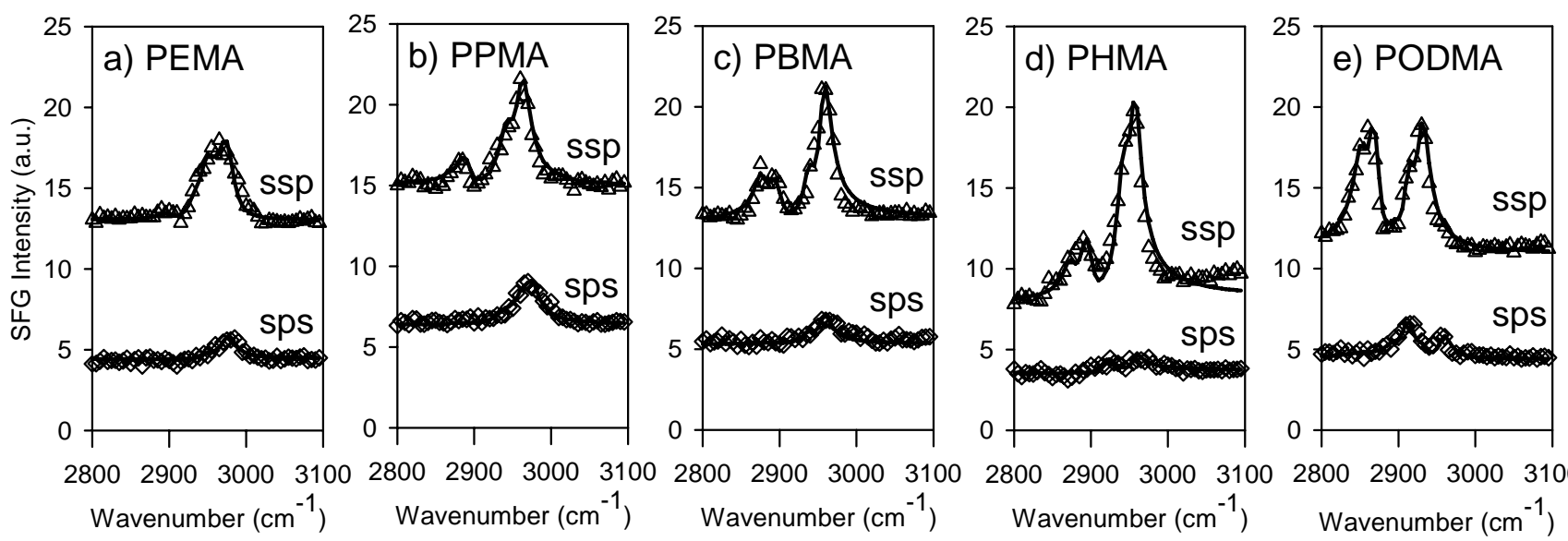

2800290030003100

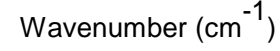

2800290030003100

Wavenumber $\left(\mathrm{cm}^{-1}\right)$

Figure 5. Measured (points) and fitted (lines) SFG spectra (ssp and sps) of PEMA, PPMA, PBMA, PHMA and PODMA in water. 


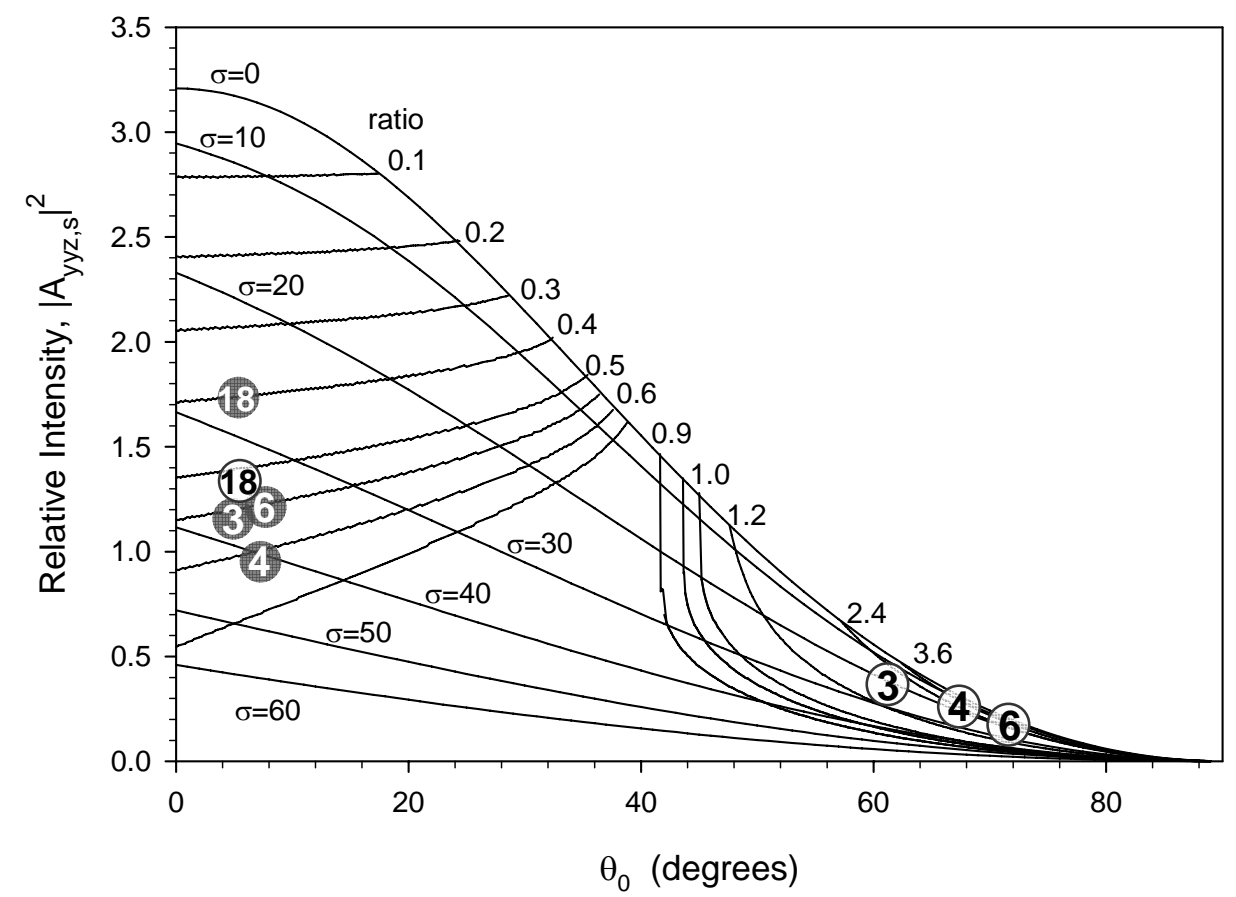

Figure 6. Relative intensity the methyl symmetric stretch as a function of $\theta_{0}, \sigma$ and $\left|\chi_{y y z, a s}\right| /\left|\chi_{\text {yzy,as }}\right|$. The intensity is relative to the ssp $\mathrm{PBMA} \mathrm{CH}_{3}$ symmetric stretch intensity in air. The points designate the values deduced for the polymer in air (shaded circles) and in water (open circles) with side chain length $\mathrm{m}(3,4,6,18)$.
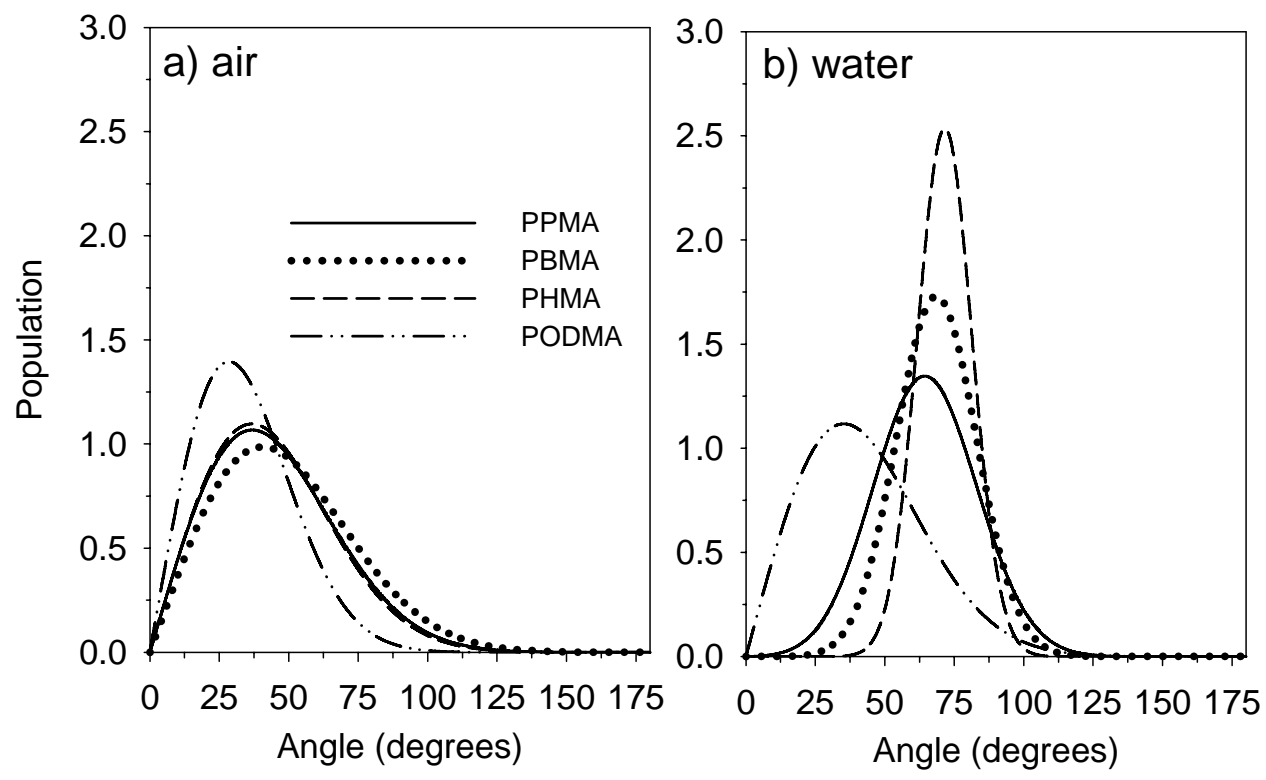

Figure 7. Gaussian orientation distributions for the side chain $\mathrm{CH}_{3}$ groups on the surfaces of PPMA, PBMA, PHMA and PODMA in (a) air and in (b) water. 
Table 1. Glass transition temperature $\left(T_{\mathrm{g}}\right)$, contact angle, surface tension, and restructuring behavior of different PAMAs. The melting point $\left(T_{\mathrm{m}}\right)$ of PODMA is also included.

\begin{tabular}{|c|c|c|c|c|c|c|c|c|}
\hline $\begin{array}{l}\text { side chain } \\
\text { length }\end{array}$ & 1 & 2 & 3 & 4 & 6 & 8 & 12 & 18 \\
\hline PAMAs & PMMA & PEMA & PPMA & PBMA & PHMA & POMA & PLMA & PODMA \\
\hline$T_{\mathrm{g}}\left({ }^{\circ} \mathrm{C}\right)^{58}$ & 108 & 65 & 40 & 20 & -5 & -20 & -65 & $\begin{array}{c}-100 \\
T_{m}=37\end{array}$ \\
\hline $\begin{array}{c}\text { Contact } \\
\text { angle in } \\
\text { water }\end{array}$ & 65.8 & 72.9 & 78.6 & 84.7 & 77.3 & 86.3 & 85.5 & 108.5 \\
\hline $\begin{array}{c}\text { Contact } \\
\text { angle in } \\
\mathrm{CH}_{2} \mathrm{I}_{2}\end{array}$ & 36.8 & 48.5 & 48.4 & 51.6 & 57.4 & 63.0 & 69.3 & 77.5 \\
\hline $\begin{array}{c}\text { Surface } \\
\text { tension } \\
(\mathrm{dyn} / \mathrm{cm}) \text { at } \\
20^{\circ} \mathrm{C} \\
\end{array}$ & 46.4 & 39.5 & 37.4 & 34.4 & 31.1 & 28.8 & 26.6 & 18.7 \\
\hline Restructure? & No & \multicolumn{4}{|c|}{ Yes } & \multicolumn{2}{|c|}{ Yes } & Yes \\
\hline Surface flat? & Yes & \multicolumn{4}{|c|}{ Yes } & \multicolumn{2}{|c|}{ No } & Yes \\
\hline Reversible? & Yes & \multicolumn{4}{|c|}{ Yes } & \multicolumn{2}{|c|}{ No } & Yes \\
\hline
\end{tabular}




\section{References}

(1) Recum, A. F. Handbook of Biomaterials Evaluation: Scientific, Technical, and Clinical Testing of Implant Materials; Macmillan Publishing Company: New York, 1986.

(2) Park, J. B.; Lakes, R. S. Biomaterials: an Introduction; Plenum Press: New York, 1992.

(3) Garbassi, F.; Morra, M.; Occhiello, E. Polymer Surfaces: from Physics to Technology; John Wiley and Sons: Chichester, 1994.

(4) Ratner, B. D.; Castner, D. G. Surface Modification of Polymeric Biomaterials; Plenum Press: New York, 1996.

(5) Feast, W. J.; Munro, H. S. Polymer Surfaces and Interfaces; John Wiley and Sons: Chichester, 1987.

(6) Feast, W. J.; Munro, H. S.; Richards, R. W. Polymer Surfaces and Interfaces II; John Wiley and Sons: Chichester, 1993.

(7) Klee, D.; Hocker, H. Adv. Polym. Sci. 1999, 149, 1-57.

(8) Angelova, N.; Hunkeler, D. Trends Biotechnol. 1999, 17, 409-420.

(9) Griffith, L. G. Acta Materialia 2000, 48, 263-277.

(10) Tirrell, M.; Kokkoli, E.; Biesalski, M. Surf. Sci. 2002, 500, 61-83.

(11) Brown, H. R. Science 1994, 263, 1411-1413.

(12) Schnell, R.; Stamm, M.; Creton, C. Macromolecules 1998, 31, 2284-2292.

(13) Edgecombe, B. D.; Stein, J. A.; Frechet, J. M. J.; Xu, Z.; Kramer, E. J. Macromolecules 1998, 31, 1292-1304.

(14) Russell, T. P. Science 2002, 297, 964-967.

(15) Khongtong, S.; Ferguson, G. S. J. Am. Chem. Soc. 2001, 123, 3588-3594.

(16) Khongtong, S.; Ferguson, G. S. J. Am. Chem. Soc. 2002, 124, 7254-7255.

(17) Langmuir, I. Science 1938, 87, 493-500.

(18) Woodruff, D.; Delchar, T. Modern Techniques of Surface Science; Cambridge Univ. Press: Cambridge, 1986.

(19) Somorjai, G. A. Introduction to Surface Chemistry and Catalysis; John Wiley and Sons: New York, 1994. 
(20) Lewis, K. B.; Ratner, B. D. J. Colloid Interface Sci. 1993, 159, 77-85.

(21) Ruckenstein, E.; Gourisankar, S. V. J. Colloid Interface Sci. 1986, 109, 557-566.

(22) Yasuda, H.; Charlson, E. J.; Charlson, E. M.; Yasuda, T.; Miyama, M.; Okuno, T. Langmuir 1991, 7, 2394-2400.

(23) Yasuda, T.; Okuno, T.; Yasuda, H. Langmuir 1994, 10, 2435-2439.

(24) Hogt, A. H.; Gregonis, D. E.; Andrade, J. D.; Kim, S. W.; Dankert, J.; Feijen, J. J. Colloid Interface Sci. 1985, 106, 289-298.

(25) Shen, Y. R. The Principles of Nonlinear Optics; John Wiley \& Sons: New York, 1984.

(26) Bain, C. D. J. Chem. Soc., Faraday Trans. 1995, 91, 1281-1296.

(27) Eisenthal, K. B. Chem. Rev. 1996, 96, 1343-1360.

(28) Zhang, D.; Ward, R. S.; Shen, Y. R.; Somorjai, G. A. J. Phys. Chem. B 1997, 101, 9060-9064.

(29) Gragson, D. E.; Richmond, G. L. J. Phys. Chem. B 1998, 102, 3847-3861.

(30) Oh-e, M.; Lvovsky, A. I.; Wei, X.; Shen, Y. R. J. Chem. Phys. 2000, 113, 8827-8832.

(31) Gautam, K. S.; Dhinojwala, A. Macromolecules 2001, 34, 1137-1139.

(32) Wang, J.; Woodcock, S. E.; Buck, S. M.; Chen, C.; Chen, Z. J. Am. Chem. Soc. 2001, 123, 94709471.

(33) Wang, J.; Paszti, Z.; Even, M. A.; Chen, Z. J. Am. Chem. Soc. 2002, 124, 7016-7023.

(34) Wilson, P. T.; Briggman, K. A.; Wallace, W. E.; Stephenson, J. C.; Richter, L. J. Appl. Phys. Lett. 2002, 80, 3084-3086.

(35) Clarke, M. L.; Wang, J.; Chen, Z. Anal. Chem. 2003, 75, 3275-3280.

(36) Chen, C.; Wang, J.; Chen, Z. Langmuir 2004, 20, 10186-10193.

(37) Chen, C. Y.; Clarke, M. L.; Wang, J.; Chen, Z. Phys. Chem. Chem. Phys. 2005, 7, 2357-2363.

(38) Gracias, D. H.; Chen, Z.; Shen, Y. R.; Somorjai, G. A. Acc. Chem. Res. 1999, 32, 930-940.

(39) Zhuang, X.; Miranda, P. B.; Kim, D.; Shen, Y. R. Phys. Rev. B: Condens. Matter 1999, 59, 1263212640 .

(40) Chen, Q.; Zhang, D.; Somorjai, G.; Bertozzi, C. R. J. Am. Chem. Soc. 1999, 121, 446-447.

(41) Chen, C.; Wang, J.; Woodcock, S. E.; Chen, Z. Langmuir 2002, 18, 1302-1309. 
(42) Chen, C.; Wang, J.; Even, M. A.; Chen, Z. Macromolecules 2002, 35, 8093-8097.

(43) Liu, Y.; Messmer, M. C. J. Phys. Chem. B 2003, 107, 9774-9779.

(44) Ye, S.; Morita, S.; Li, G. F.; Noda, H.; Tanaka, M.; Uosaki, K.; Osawa, M. Macromolecules 2003, 36, 5694-5703.

(45) Morita, S.; Ye, S.; Li, G. F.; Osawa, M. Vib. Spectrosc. 2004, 35, 15-19.

(46) Johnson, W. C.; Wang, J.; Chen, Z. J. Phys. Chem. B 2005, 109, 6280-6286.

(47) Kweskin, S. J.; Komvopoulos, K.; Somorjai, G. A. Langmuir 2005, 21, 3647-3652.

(48) Wang, J.; Chen, C.; Buck, S. M.; Chen, Z. J. Phys. Chem. B 2001, 105, 12118-12125.

(49) Wei, X.; Zhuang, X.; Hong, S.; Goto, T.; Shen, Y. R. Phys. Rev. Lett. 1999, 82, 4256-4259.

(50) Wilk, D.; Johannsmann, D.; Stanners, C.; Shen, Y. R. Phys. Rev. B: Condens. Matter 1995, 51, 10057-10067.

(51) Lambert, A. G.; Neivandt, D. J.; Briggs, A. M.; Usadi, E. W.; Davies, P. B. J. Phys. Chem. B 2002, 106, 5461-5469.

(52) McGall, S. J.; Davies, P. B.; Neivandt, D. J. J. Phys. Chem. B 2004, 108, 16030-16039.

(53) Clarke, M. L. Interactions of Polymers and Proteins at Interfaces Studied by Sum Frequency Generation Vibrational Spectroscopy, Ph.D. Dissertation, University of Michigan, Ann Arbor, 2006.

(54) Nolin, B.; Jones, R. N. Can. J. Chem. 1956, 34, 1392-1404.

(55) Wang, J.; Castonguay, M.; Roy, J.; Zahidi, E.; McBreen, P. H. J. Phys. Chem. B 1999, 103, 43824386.

(56) Stanners, C. D.; Du, Q.; Chin, R. P.; Cremer, P.; Somorjai, G. A.; Shen, Y.R. Chem. Phys. Lett. 1995, 232, 407-413.

(57) Sefler, G. A.; Du, Q.; Miranda, P. B.; Shen, Y. R. Chem. Phys. Lett. 1995, 235, 347-354.

(58) Andrade, J. D. Polymer Surface Dynamics; Plenum Press: New York, 1998. 
